\title{
1-YIA-07 YIA
}

\section{Deficiency of cardiac natriuretic peptide signaling promotes peripartum cardiomyopathy-like remodeling in the mouse heart}

\author{
Takeshi Tokudome $^{1}$, Kentaro Otani ${ }^{2}$, Chizuko Kamiya ${ }^{3}$, Mikiya Miyazato ${ }^{1}$, Kenji Kangawa $^{4}$ \\ ${ }^{I}$ Dept. Biochemistry., NCVC, ${ }^{2}$ Dept. Regenerative Medicine-Tissue Engineering., NCVC, ${ }^{3}$ Div. Perinatology- \\ Gynecology., NCVC, ${ }^{4} \mathrm{NCVC}$
}

Background. The maternal circulatory system changes dynamically during perinatal period. Although atrial and brain natriuretic peptides (ANP and BNP, respectively) produced in the heart control circulatory homeostasis through their common receptor, NPR1, the pathophysiological roles of endogenous ANP/BNP in the perinatal period are not fully understood. Methods. To clarify the pathophysiological roles of the endogenous ANP/BNP-NPR1 system during the perinatal period, the phenotype of female wild-type and conventional or tissue-specific Npr1-knockout mice during the perinatal period was examined. Results. In wild-type mice, lactation but not pregnancy induced reversible cardiac hypertrophy accompanied by increases in fetal cardiac gene mRNAs and ERK1/2 phosphorylation. Npr1-knockout mice exhibited significantly higher plasma aldosterone level than did wild-type mice, severe cardiac hypertrophy accompanied by fibrosis, and left ventricular dysfunction in the lactation period. In the hearts of Npr1-knockout mice during or after the lactation period, an increase in interleukin-6 mRNA expression, phosphorylation of STAT3, and activation of the calcineurin-NFAT pathway were observed. Pharmacological inhibition of the mineralocorticoid receptor or neuron-specific deletion of the mineralocorticoid receptor gene significantly ameliorated cardiac hypertrophy in lactating Npr1-knockout mice. Anti-interleukin-6 receptor antibody administration tended to reduce cardiac hypertrophy in lactating Npr1-knockout mice. Conclusions. These results suggest that the endogenous ANP/BNP-NPR1 system plays an important role in protecting the maternal heart from interleukin-6-induced inflammation and remodeling in the lactation period, a condition mimicking peripartum cardiomyopathy. (Circulation, in press) 